\title{
LA DEMOCRACIA MODERNA \\ Y LA DISTINCIÓN DE MORAL \\ Y DERECHO \\ (CON ESPECIAL ATENCIÓN A J. RAWLS)
}

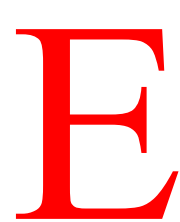

$\mathrm{n}$ general pienso que los dos mil años (aproximadamente) que separan a la democracia moderna de la antigua son más que suficientes para suponer que tras la identidad de vocablo se esconde una profunda diferencia de significación. En particular una diferencia que es comúnmente admitida, y desde luego muy decisiva, es la de que la democracia antigua no tenía propiamente representantes. Tenía desde luego funcionarios; pero la brevedad de los mandatos, la exigencia de responsabilidades después de concluidos, el sorteo a veces de los cargos.... todo indica que actuaban como meros delegados o comisionados por parte del pueblo. Una diferencia también clara, y que nos interesa más directamente, es la que se refiere a las relaciones entre la moral y el Derecho. Basta abrir la Ética a Nicómaco de Aristóteles para encontrarse en las primeras páginas con la sorprendente afirmación (sorprendente para un moderno), de que se trata de una investigación política; y esto porque, como había dicho anteriormente, «de todas las ciencias la política es la soberana y arquitectónica o directiva por antonomasia». Bastantes años antes (el 399 a.C) el texto de la acusación contra Sócrates decía: «Es Sócrates reo del delito de no reconocer los dioses que el Estado reconoce y de introducir otros genios o espíritus extraños, y asimismo de corromper a nuestros jóvenes». Como se ve, una acusación difícil de imaginar en una democracia moderna.

La teoría de la distinción entre la moral y el Derecho se abre paso a fines del siglo XVII y principios del XVIII, con Bayle, Locke y Thomasius, impulsada por el problema de la tolerancia religiosa. Se siente la necesidad de liberar a la religión del control y la coacción del Estado, y el principal argumento que se encuentra es que la religión consiste esencialmente en actos de convicción y de voluntad, por lo que la intervención del Estado lo más que podría lograr serían conversiones exteriores, es decir, actos de hipocresía. Como se argumenta 
filosóficamente, la religión que se tiene en cuenta no es primordialmente la revelada, sino la que dicta la razón, es decir, la religión natural, que no es más que una parte de la moral, la que se refiere a nuestros deberes para con Dios. Así es como resulta que lo que en definitiva se diferencia del Derecho (bajo el control del Estado) es la moral en general, y no sólo la religión.

Rousseau no va a apoyar esta postura. Con su defensa de la «religión civil» pone de manifiesto que también en este punto, en cuestiones de opinión, vuelve sus ojos a la democracia antigua.

Pero sí va a seguir esa dirección de Kant, que la consolida y depura, al poner el énfasis en que la moral es cuestión de actitud o disposición de la voluntad; por eso es esencialmente interior y exenta de coacción, a la inversa que el Derecho.

Las dos revoluciones que dan comienzo a la democracia moderna reconocen, implícitamente, la distinción de moral y Derecho, al admitir la libertad, o al menos tolerancia, de las opiniones, especialmente de las religiosas; pero incluso en la norteamericana éstas están consideradas bajo el punto de vista de la razón, la convicción y la conciencia; por lo que, al menos implícitamente, se reconoce que el mismo principio será aplicable a todas las actitudes que procedan de la razón, de la convicción y de la conciencia ${ }^{1}$.

Con Hegel la teoría de la diferenciación entre la moral y el Derecho se complica. Por un lado, parte de la caracterización de ambos hecha por Kant; pero, por otro, le importa mucho la determinación concreta de lo que es justo y lo que es bueno, lo que hay que hacer en cada caso; y esto no le parece fácil más que a través de las instituciones, leyes y costumbres del Estado. En definitiva la moral y el Derecho se dan juntos en lo que se practica generalmente con convicción o adhesión, satisfaciendo así al lado objetivo, del Derecho, y al subjetivo, de la moral: en la Sittlichkeit. Esto es lo que está llegando a su pleno desarrollo en los Estados modernos, es decir, en los democráticos recién instaurados.

Esa es la visión idealista, e idealizada, de la democracia. Desde una perspectiva realista, de observación de la realidad, veremos que esa concordancia no está garantizada. Pero antes veamos nuevas complicaciones teóricas para la distinción de moral y Derecho. Me refiero al utilitarismo de Bentham. Las complicaciones vienen también aquí por el lado objetivo, de la determinación del contenido, de lo que se ha de hacer, de lo bueno. Si bien esa determinación no corre ahora a cargo de las instituciones, leyes y costumbres ya establecidas

${ }^{1}$ Cfr. Declaración de derechos del buen pueblo de Virginia (1776), XVI: Enmiendas a la Constitución de EE.UU. (1791), 1. ${ }^{\mathrm{a}}$ Enmienda, Declaración de derechos del hombre y del ciudadano (de la Asamblea francesa, 1789), art. 10. 
en el Estado, sino de la razón, que calcula las consecuencias, el resultado, en términos de aumento o disminución de la felicidad o bienestar de los afectados. Pero esto es lo mismo que tiene que hacer la política en cuanto determina el contenido del Derecho. Y Bentham se fijó tanto en eso, que descuidó, «dejó en blanco», según la expresión de Mill, una de las dos partes en que «consiste la moralidad»; atendió sólo a «la regulación de las acciones exteriores» (las que tienen más trascendencia directa para la política y el Derecho); pero no al otro aspecto, de la «autoeducación, de la formación del ser humano, de sus afectos y de su voluntad». $\mathrm{Y}$, sin acceder a este aspecto, no parece posible distinguir adecuadamente entre la moral y el Derecho.

A costa de su propia experiencia personal aprendió Mill a valorar la importancia de este otro aspecto; y la contribución a su mejora le pareció el elemento más decisivo para valorar positivamente el régimen representativo, o democrático en el sentido moderno. Pero no basta con que sea democrático: ha de ser también liberal, en el sentido ideológico o político del término. De aquí los esfuerzos de Mill por asegurar una esfera autónoma de la personalidad, sustraída al poder de la colectividad, tanto política como social. El principio democrático solo no basta, porque «el pueblo que ejerce el poder no es siempre el mismo pueblo sobre el cual es ejercido; y el «gobierno de sí mismo», de que se habla, no es el gobierno de cada uno por sí, sino el gobierno de cada uno por todos los demás. En realidad, la voluntad del pueblo significa, prácticamente, la voluntad de la porción más numerosa o más activa del pueblo; de la mayoría o de aquellos que logran hacerse aceptar como tal» ${ }^{2}$.

Pocos años antes, Tocqueville había mostrado en la realidad de la democracia norteamericana este poder de la colectividad ejerciéndose sobre los individuos. Llama especialmente la atención sobre el hecho de que ese poder no se ejerce sólo sobre los cuerpos, materialmente, sino también directamente sobre las almas, en las opiniones o creencias, en las actitudes de la voluntad. La base está en la autoridad de la mayoría, y ésta a su vez se apoya en que las sociedades igualitarias, que han dejado de tener respeto por la autoridad de individuos particulares, lo trasfieren al conjunto, a la gran masa, al público en general. Esta postura se ve reforzada, por un lado, en virtud del principio (utilitarista) de que se ha de dar la preferencia a los intereses del mayor número; y, por otro, gracias a un trasvase u ósmosis del principio del gobierno de la mayoría, que rige en la democracia política, a ese otro terreno de la democracia social, en que se extiende también a las actitudes y opiniones. Estos refuerzos

2 J. S. Mill, On Libery, cap. I, en la traducción de P. de Azcárate, Madrid (Alianza), 1970, págs. 58-59. 
determinan, al menos en parte, el modo como la mayoría se impone: un modo poco apropiado, al menos desde el punto de vista de un liberal, para la moral: «No persuade de sus ideas, las impone y las hace penetrar en las almas por una especie de presión inmensa del espíritu de todos sobre la inteligencia de cada uno» ${ }^{3}$.

Tenemos, por tanto, que la realidad democrática, sobre todo esa realidad que resulta de aplicar en la sociedad (en general) los criterios propios de la política, supone una dificultad, al menos práctica, en los hechos, aun cuando no en los principios, para la diferenciación de la moral y el Derecho. Podríamos precisar más, diciendo que no en los principios del propio Tocqueville; no se trata de una posición teórica de éste, sino de la realidad que él expone. Ahora bien, en cuanto que esta realidad abarca las opiniones, tal vez se pueda decir que éstas implícitamente están suponiendo unos principios contrarios a la distinción.

No es lo mismo en el caso de J. Rawls. Por muy importantes que sean para él las opiniones de las gentes, lo que pretende primordialmente no es exponerlas, sino en todo caso tenerlas en cuenta, partir de ellas; lo que primordialmente pretende es exponer su propia teoría: Una teoría de la justicia, según el título de su obra principal (1971). Pero aquí ya empiezan las ambigüedades y dificultades para la distinción de moral y Derecho. Desde luego no se trata de una teoría de la moral en general, de una ética completa o general, sino de una teoría de la justicia; pero tampoco de toda la justicia, sino, según la terminología (que se presta a confusión en nuestro ambiente) de la obra principal, sólo de la justicia «social»; más en concreto, de la «estructura básica de la sociedad»; o, «más exactamente», según sus propias palabras, el objeto primordial de su obra es «el modo en que las principales instituciones sociales distribuyen los derechos y deberes fundamentales y determinan la distribución de las ventajas de la cooperación social» ${ }^{4}$. Por principales instituciones sociales entiende «la constitución política y los arreglos o disposiciones (arrangements) económicos y sociales fundamentales». Como ejemplos cita «la protección jurídica de la libertad de pensamiento y de conciencia, el mercado libre, la propiedad privada de los medios de producción y la familia monógama». Fuera de la protección de la libertad de pensamiento y de conciencia, no se ve, pues, si las otras instituciones tienen carácter jurídico o se pretende que lo tengan (y hasta qué grado). En realidad no se ve siquiera a qué sociedad se está refiriendo. Al advertir que se trata de «un sistema cerrado aislado de las otras sociedades», parece que se refiere a las sociedades que se corresponden con los Estados.

${ }^{3}$ A. de Tocqueville, De la démocratie enAmerique, II, 1. a parte, cap. II. 
Pero en un artículo del año 1978, especialmente dedicado a este tema de la «estructura básica», y que no pretende modificar, sino ampliar la exposición de la obra principal $^{5}$, son «las naciones» el ejemplo ilustrativo de «una sociedad entendida como esquema mas o menos autosuficiente de cooperación social y en posesión de una cultura más o menos completa ${ }^{6}$. Ahora bien, todos sabemos que las naciones no se corresponden necesariamente con los Estados. Además en la explicación de la «estructura básica» se advierte que «un régimen económico no es sólo un esquema institucional para la satisfacción de los deseos y aspiraciones existentes, sino un modo de configurar esos deseos y aspiraciones para el futuro». Y, de un modo más general, que «la estructura básica configura el modo en que el sistema social produce y reproduce a lo largo del tiempo una cierta forma de cultura» ${ }^{7}$. Todo esto pone más de manifiesto que no se trata sólo de instituciones jurídicas o que se pretende que lo sean, que reciban la protección, particular o específica, del Derecho.

En los trabajos de los años ochenta la realidad que aparece como referencia de la categoría o concepto de sociedad como «sistema cerrado y autosuficiente de cooperación» es la de un «Estado-nación» ${ }^{8}$. En cuanto a la expresión «justicia social», alterna con la de «justicia social y política», o bien es sustituida simplemente por la de «justicia política»; e incluso la de «estructura básica de la sociedad cede el paso a las de «estructura básica de un régimen constitucional democrático», o de «estructura básica de un régimen constitucional», o «de una democracia constitucional moderna», o «de un régimen democrático ${ }^{9}$. Pero esto no quiere decir que se haya reducido definitivamente su aplicación al campo de lo político; y mucho menos que se haya precisado el alcance de éste con una diferenciación, o definición, de lo político, de la categoría de lo político. La prueba de ello es, en primer lugar, que se sigue empleando, aunque con mucha menos frecuencia, la expresión de «estructura básica de la sociedad»; y, sobre todo, que explícitamente se sigue sosteniendo que se trata de las «instituciones políticas, sociales y económicas» ${ }^{10}$, o de las «principales instituciones políticas y

${ }^{5}$ J. Rawls, «The Basic Structure as Subject», en A. I. Goldman-J. Kim (eds.): Values and Morals, Dordrecht (Reidel), 1978, págs. 47-71 (Cfr. especialmente a este respecto pág. 69, nota 1).

${ }^{6}$ J. Rawls, Op. cit., (nota ant.), pág. 70 , nota 8.

${ }^{7}$ J. Rawls, Op. cit., pág. 55.

8 J. Rawls, «Kantian Constructivism in Moral Theory», en The Journal of Philosophy, LXXVII (1980), en J. Rawls, Justicia como equidad, traducción de M. A. Rodilla (Tecnos), 1986, págs. 145 y 155.

${ }^{9}$ J. Rawls, «Justice as Fairness: Political not Metaphysical», Philosophy and Public Affairs, 14/3 (1985), págs. 224-225; «The Priority of Right and Ideas of the Good», en Phylosophy and Public Affairs, 17/4 (1988), págs. 252 y $261-2$.

${ }^{10}$ J. Rawls, «Justice as Fairness...» (cit.), págs. 224-225. 
sociales ${ }^{11}$. Por consiguiente, no hay una verdadera diferenciación de lo político, y no digamos de lo jurídico.

En cuanto a la categoría o concepto de la moral, o de lo moral, vamos a ver que no sólo no se da en Rawls su delimitación o precisión, sino que además se hace imposible, o muy difícil, establecerla, al menos en un sentido similar al que tenía en la Ilustración, y particularmente en Kant.

Lo primero que vamos a considerar es el destinatario o sujeto a quien va dirigida la doctrina o teoría. No se trata de un tema que Rawls exponga expresamente (como el anterior de la estructura básica), pero de las fórmulas repetidas sí se desprende con claridad que se tiene en cuenta esta cuestión del destinatario, o de dónde ha de ser recibida o aceptada esa doctrina o teoría. Esas fórmulas o expresiones no son uniformes. Así encontramos la de «un Estado democrático moderno», «un Estado constitucional democrático», «una democracia constitucional», «un régimen democrático», «un régimen constitucional»; pero junto a ellas la de «para una sociedad democrática» aparece también repetida, y a veces muy próxima a una de las anteriores. Esto sugiere lo mismo que veíamos en el apartado anterior: que puede haber en la concepción de Rawls un predominio de lo político (acentuado todavía más, con respecto a la obra principal, en los trabajos de los años ochenta); pero que en ningún caso hay exclusión de lo meramente social (en cuanto contrapuesto a lo político). Por lo demás, hay una indudable conexión entre este tema y el anterior: las ideas son para un Estado o una sociedad democráticos, para que estos organicen sobre ellas, y con ellas, su estructura básica.

Rawls no se preocupa por fijar exactamente los límites de esa «sociedad democrática», o ese «Estado democrático moderno». Por el modo como se expresa («desde, digamos, la Declaración de Independencia»), parece que se refiere primordialmente al Estado o sociedad que surgen de las revoluciones de finales del siglo XVIII, y sobre todo en Norteamérica. Pero desde luego sin que eso suponga un aislamiento o separación, ni desde el punto de vista histórico ni desde el geográfico. Las conexiones más directas son con la época que arranca del Renacimiento y con los países europeos. Así se desprende de la descripción que hace de los orígenes de las «condiciones sociales e históricas de ese Estado»: en las guerras de religión que siguieron a la Reforma (protestante) y el subsiguiente desarrollo del principio de tolerancia, así como en el desenvolvimiento del Gobierno constitucional y de las instituciones de la economía de mercado industrial ampliado.

${ }^{11}$ J. Rawls, «The Priority of Right...» (cit.), pág. 269. 
Estas condiciones «afectan profundamente a los requisitos» de lo que Rawls va buscando. Lo que va buscando, por lo que sabemos hasta aquí, es una teoría de la justicia que se califica de «social», o de «política», porque se refiere a la estructura básica de la sociedad, o Estado, y va dirigida a la sociedad democrática, o Estado democrático moderno. Pero ahora vamos a ver otra característica de esa teoría que se busca. Se trata de encontrar unos «principios de justicia que han de servir como principios de justicia públicos y efectivos»; «un acuerdo público y viable en materias de justicia social»; «un acuerdo sobre una concepción de la justicia viable destinada a conseguir una suficiente convergencia de opinión»; «un procedimiento públicamente establecido y mutuamente aceptable»; «un punto de vista públicamente reconocido»; «una base viable y compartida para una concepción política de la justicia»; «una concepción pública de la justicia que sea viable, es decir, que sirva como una base pública de justificación, de modo que se pueda esperar razonablemente que los ciudadanos la aceptarán». Estamos, pues, ante un nuevo sentido en que la justicia propugnada por Rawls es social o política (además de referirse a la estructura básica de la sociedad y de estar orientada a la sociedad democrática): es una justicia que tiene que ser pública, o socialmente, reconocida, generalmente aceptada o compartida. No se basa en razones o argumentaciones que se puedan alegar desde un punto de vista teórico mejor o peor fundado, sino en un «punto de vista social». «Es el punto de vista públicamente compartido por los ciudadanos de una sociedad bien-ordenada, y los principios que provienen de él son aceptados por ellos como dotados de autoridad con respecto a las pretensiones de los individuos y asociaciones ${ }^{12}$. Por consiguiente, «las pretensiones de la justicia como equidad (su propia teoría), en cuanto que es una concepción política, son prácticas y no metafísicas o epistemológicas. Es decir, no se presenta como una concepción de la justicia que sea verdadera, sino como una concepción que pueda servir de base para el entendimiento informado y voluntario entre los ciudadanos considerados como personas libres e iguales ${ }^{13}$.

En las condiciones de la sociedad democrática moderna (a que anteriormente nos hemos referido) ninguna de las doctrinas éticas tradicionales está en condiciones de afrontar esa prueba de servir de base pública y generalmente reconocida a una concepción de la justicia. ¿Razones? La primera es que se trata de concepciones «generales», que se refieren a todas las actuaciones humanas (y no sólo a la estructura básica de la sociedad). Así el utilitarismo, por ejemplo, se

${ }^{12}$ J. Rawls, «Kantian Constructivism...» (cit. nota 8), pág. 185.

${ }^{13}$ J, Rawls, «Justice as Fairness...» (cit. nota 9), pág. 230. 
refiere tanto a las acciones de los individuos como al Derecho que rige entre las naciones. La segunda es que son «comprensivas», es decir, se refieren a otros aspectos de la vida humana (y no sólo al socio-político). Abarcan concepciones de lo que es valioso en la vida humana, ideales de virtud, y otros, que tratan de conformar la vida humana con mayor o menor amplitud, en último término en toda su amplitud. La tercera, menos expresamente formulada por Rawls, pero que se manifiesta sobre todo por contraste con su propia posición, es que esas doctrinas éticas tradicionales razonan a partir de sus propios principios, tratando de convencer de ellos, o desde ellos, dándoles por supuestos.

En estos tres aspectos se diferencia la postura de Rawls de las doctrinas éticas tradicionales. Los dos primeros (limitación a la justicia en cuanto estructura básica de la sociedad, y al sector o aspecto socio-político en la democracia moderna) ya los hemos visto con anterioridad. Veremos, pues, ahora el tercero. Desde luego Rawls no se limita a recoger las convicciones generalizadas que encuentra ya formuladas expresamente en la sociedad democrática. Parte más bien de lo que está implícito en la «cultura pública» de esa sociedad y elabora a partir de eso los principios que espera sean compartidos una vez conocidos. El principal supuesto de que se parte es la concepción «de la sociedad como un sistema equitativo de cooperación entre personas libres e iguales» ${ }^{14}$.

«Los ciudadanos afirman su concepción pública de la justicia porque casa con sus convicciones consideradas (o ponderadas) y es coherente con el tipo de persona que, tras la debida reflexión, desean ser». Nos dirigimos a la «cultura pública de una sociedad democrática» y se «espera hacer consciente una concepción de la persona y de la cooperación social que se conjetura que está implícita en esa cultura, o al menos que es congenial con sus tendencias más profundas, cuando se las expresa y presenta de forma apropiada» ${ }^{15}$.

Esta operación de recogida o aprehensión de las opiniones explícita o implícitamente compartidas no tiene porqué estar enfrentada con las diversas concepciones morales, religiosas o filosóficas vigentes en la sociedad. Es más bien de ellas de donde se recogen. Pero no de ellas en cuanto consideradas en su totalidad, en todas sus partes, sino tan solo en cuanto coinciden: dejando a un lado sus diferencias, se toma tan solo aquella parte (referente a la concepción socio-política de la estructura básica de la sociedad) en que coinciden. Se trata, pues, de «identificar el núcleo de un consenso por superposición, es decir, las ideas intuitivas compartidas que, elaboradas

${ }^{14}$ J. Rawls, «Justice as Fairness...», pág. 231.

${ }^{15}$ J. Rawls, «Kantian Constructivism...», pág. 183 
hasta formar una concepción política de la justicia, resultan ser suficientes para sustentar un régimen constitucional» ${ }^{16}$. En cuanto las doctrinas filosóficas no entran a formar parte de ese consenso, se las deja a un lado, como viene ya haciéndose en materia política con las confesiones religiosas. Esto es lo que Raw1s califica como «aplicar el principio de tolerancia a la filosofía misma» ${ }^{17}$.

Ya se comprenderá, por todo lo que llevamos expuesto últimamente, la dificultad o imposibilidad a que aludíamos de que Rawls pudiera coincidir con la Ilustración, y en concreto con Kant, en la caracterización de la moralidad. El mismo viene a reconocerlo. A pesar de su continuado esfuerzo por seguir presentándose como kantiano, las diferencias, en este punto esencial, se hacen demasiado patentes: «aunque considero la concepción de la justicia como equidad como una concepción kantiana, difiero de la doctrina de Kant en importantes aspectos. Hago notar aquí que la concepción de la justicia como equidad asigna cierta primacía a lo social: esto es, el primer objeto de la justicia es la estructura básica de la sociedad, y los ciudadanos tienen que llegar en primer lugar a un entendimiento público sobre una concepción de la justicia aplicable a este objeto... En contraste con ello, el tratamiento kantiano del Imperativo Categórico se aplica a las máximas personales de individuos sinceros y conscientes en la vida diaria» ${ }^{18}$.

Aun cuando las últimas palabras («en la vida diaria»), puedan desviar la atención hacia la diferencia de objeto (expresado, a mi entender, de modo poco apropiado en el caso de Kant), a través de las anteriores se trasluce toda la importancia de la diferencia, de carácter, y no sólo de objeto, entre la concepción de Rawls (de la justicia) y la visión kantiana (de la moralidad). Desde el punto de vista kantiano no es que se «aplique la tolerancia a la filosofía», sino que se aplica a todas las opiniones, en el sentido de no imponer ninguna, sino aceptar simplemente la que se imponga por sí misma, por la vía de la convicción. Desde ese punto de vista, moral, las opiniones no son válidas más que en cuanto uno se las apropia, es decir, son propias o apropiadas, personales, en cuanto que son convicciones. No tiene sentido tratar de imponerlas: sólo se forman o se crean por razones, atendiendo a razones. El punto de vista de Rawls, en cambio, como hemos visto, es social o público, es colectivo. Desde luego no es que por eso se trate de imponer las ideas u opiniones por la fuerza, o la coacción; no, ese no sólo no es un procedimiento apropiado para crear convicciones, sino que tampoco lo es para dar

${ }^{16}$ J. Rawls, «Justice as Fairness...», págs. 246-247.

${ }^{17}$ J. Rawls, Op. cit. (nota ant.), pág. 223.

${ }^{18}$ J. Rawls, «Kantian Constructivism...», pág. 169. 
origen a opiniones colectivas. Pero hay otras maneras de imponer o hacer prevalecer las ideas. Rousseau previó, para los que no fueran capaces de compartir los «sentimientos de sociabilidad» ${ }^{19}$ de su «religión civil», una solución sencilla: la pena de destierro.

- ¿Cuál es la solución de Rawls a este respecto?

-Desde luego, por lo pronto, dar la preferencia a su propia doctrina sobre cualquier otra concepción. Se supone que las personas tienen ciertas convicciones morales; pero en caso de conflicto han de prevalecer sobre esas convicciones los «principios de justicia» reconocidos. Por lo demás, no es necesario revisar esas convicciones ni renunciar a ellas cuando no están cubiertas o apoyadas por los «principios». «Lo esencial es que, cuando personas con diferentes convicciones tienen pretensiones contrapuestas sobre la estructura básica en cuestiones de principio político, han de juzgar y sentenciar sobre esas pretensiones de acuerdo a los principios de justicia» ${ }^{20}$. Dicho de otra manera, «no es que haya que evitar o eliminar las ideas del bien (o acerca de lo que es bueno), lo cual es imposible. De lo que se trata más bien es de que las ideas que se apliquen tienen que ser políticas: tienen que estar adaptadas (tailored) para satisfacer las restricciones impuestas por la concepción política de la justicia y encajar en el espacio que ella permite» ${ }^{21}$.

- ¿ ¿cuál es el fundamento de tal preferencia?

-Desde luego parece que el carácter socio-político de la solución adoptada, en el triple sentido de ese carácter: referirse a la estructura básica de la sociedad, estar orientada a la sociedad democrática moderna y estar apoyada sobre las opiniones pública o socialmente compartidas, aun cuando pueda ser que sólo implícitamente. En otros términos, pudiéramos decir que lo que da la preeminencia a la propia postura, a la postura de Rawls, es su carácter «democrático»: orientarse a la determinación de la estructura básica de la sociedad democrática moderna y apoyarse en una solución democrática. Pero, entiéndase bien, el sentido de la «democracia» a que aquí nos estamos refiriendo no se restringe al campo propiamente político (la estructura del Estado y las soluciones adoptadas de acuerdo a la constitución política): Rawls puede dar preferencia a ese sector de lo estatal, pero no reduce su teoría a ese sector; entre otras razones porque no lo delimita con respecto al resto de los sectores sociales, con respecto a lo meramente social. Esta falta de delimitación o de definición favorece ese trasvase u ósmosis de los criterios aplicables en la democracia política propiamente dicha al terreno de la democracia

${ }^{19}$ J. J. Rousseau, Du Contrat social, IV, 8.

${ }^{20}$ J. Rawls, A Theory of Justice (cit. nota 4), págs. 220-221.

${ }^{21}$ J. Rawls, «The Priority of Right...» (cit. nota 9), pág. 271. 
social, en que se aplica también a las actitudes y opiniones, a que nos referíamos ya al hablar de Tocqueville. Ese trasvase se podría evitar, al menos teóricamente (negar su legitimidad) con una adecuada diferenciación de la moral, no sólo con respecto al Derecho, sino también con respecto a los usos sociales. Pero precisamente esa diferenciación es la que Rawls no está en condiciones de poder hacer: con su postura, tal como se ha perfilado sobre todo en los trabajos de los años ochenta, se la ha hecho imposible.

Sin embargo, creo que sería grave error pensar que la moral, en el sentido de algo personal, de convicción, está totalmente ausente de la teoría de Rawls. Tiene que estar de algún modo presente, porque lo está, aun cuando no de una manera claramente diferenciada, en la «cultura pública» de la que él parte, o en la que él fundamentalmente se apoya, y, más en concreto, en la concepción de las personas que han de cooperar en la sociedad como un sistema equitativo. En efecto, Rawls afirma expresamente que las personas de tal sociedad, que han de formar tal sociedad, «se consideran así mismas como personas morales». Lo cual significa, en primer lugar, que tienen «la capacidad para un sentido de la justicia efectivo, esto es, la capacidad para entender, aplicar y actuar a partir de (y no meramente de acuerdo con) los principios de la justicia». No parece caber duda que este «sentido de la justicia» tiene que ser algo personal, propio de cada uno, referente a la capacidad y actuación de cada uno. Que, por consiguiente, el término de «morales», que se atribuyen a las personas y que es desarrollado o explicado por medio de ese «sentido de la justicia», que se afirma que tienen, o al menos la capacidad para él, ha de entenderse mucho más en conexión con la idea kantiana de moral, que con la concepción de Rawls de la justicia social o política en lo que ésta tiene de más característico o destacado, es decir, su carácter consensual o socio-político.

Por otro lado, el acuerdo sobre los principios de justicia que han de regir la estructura básica «ha de ser adoptado, como cualquier otro, bajo condiciones apropiadas. En especial, estas condiciones apropiadas. En especial, estas condiciones tienen que colocar a las personas libres e iguales en una situación equitativa y no pueden permitir que algunas hagan mejor negocio que otras. Además han de quedar excluidas las amenazas de fuerza y coacción, así como el engaño y el fraude». Las partes de ese acuerdo han de concebirse, pues, como «situadas equitativamente (fairly) y en disposición de adoptar un convenio sometido a las adecuadas restricciones en cuanto a lo que han de contar como buenas razones ${ }^{23}$. Y no parece nada secundario que el concepto que sirve a Rawls para designar de preferencia

${ }^{23}$ J. Rawls, «Justice as Fairness...», págs. 235 y 239. 
su propia teoría, el concepto de equidad (fairnes) o de equitativo (fair, fairly), no sea objeto de acuerdo o consenso, sino que pertenezca a las condiciones necesarias para que ese acuerdo sea válido. Esto lo libera de las características de la justicia propiamente sociopolítica, y de nuevo nos encontraríamos con que estamos más bien en el terreno de la moral entendida en el sentido tradicional (kantiano). Esto se pone más de manifiesto cuando Rawls alude a que un procedimiento neutral (en un sentido que corresponde a la justicia como equidad) puede decirse que es un procedimiento «justificado mediante la apelación a valores neutrales, es decir, a valores tales como la imparcialidad, la coherencia en la aplicación de los principios generales a todos los casos que se encuentren razonablemente relacionados..., la igualdad de oportunidades para que las partes en conflicto presenten sus alegaciones, y otros por el estilo. Se trata de valores que regulan procedimientos equitativos (fair) para decidir entre las pretensiones en conflicto» ${ }^{24}$.

A la vista de estos textos, creo que se ha de considerar por lo menos engañosa la interpretación que contrapone simplemente las concepciones filosóficas, por un lado, y la «mera descripción histórico-sociológica del modo en que actualmente vivimos», por otro ${ }^{25}$. En medio de ambos, o, si se prefiere, dentro del segundo aspecto, están esos presupuestos, esas condiciones, esos valores que son previos o independientes del «arreglo con éxito» entre los individuos, a que parece que quiere reducirse nuestro actual «modo de vivir». Esos presupuestos, condiciones y valores, aparte de, prescindiendo de, su fundamento filosófico, están presentes también en nuestro actual modo de vivir: forman parte de nuestras tradiciones todavía vivas y de las respuestas que se han ido dando, en nuestra sociedad, a los problemas que continúan siendo los nuestros ${ }^{26}$.

Sería lamentable abandonar la consideración de esos elementos, que forman también parte de nuestro actual modo de vivir, porque

${ }^{24}$ J. Rawls, «The Priority of Right...», pág. 261.

${ }^{25}$ Así R. Rorty, «The priority of democracy to philosophy», en Objetivity, relativism, and truth - Philosophical Papers, I, Cambridge (University Press), 1991, pág. 185. Los textos de Rawls últimamente citados son respectivamente de 1985 y 1988, y el trabajo de Rorty se había publicado por primera vez en este último año, en M. Peterson-R. Vaughan (eds.), The Virginia Statute of Religious Freedom, Cambridge (University Press), 1988; pero se había expuesto previamente en una conferencia dada en la Universidad de Virginia en 1984; Rawls, por lo tanto, podía conocer o tener referencias de esta interpretación; en este caso los textos citados podrían tener el sentido de respuesta, lo que les daría un mayor valor. La interpretación de Rorty ha sido criticada por K. O. Apel, Diskurs und Verantwortung - Das Problem des Übergans zur Postkonventionellen Moral, Frankfurt/M. (Suhrkamp), 1988, págs. 404 y ss.

${ }^{26}$ El propio Rorty alude también a esto: $O p$. cit., pág. 184. 
sea más novedoso, más atractivo y más fácil de fijarse en el aspecto de los convenios, acuerdos, consensos o arreglos. En realidad éstos dependen de aquéllos, no sólo en cuanto a su validez, sino también en cuanto a su consistencia y efectividad; porque éstas se apoyan en la actuación de los individuos, y esa actuación responde a actitudes, sentimientos, estados de ánimo más o menos personales, más o menos individualizados. El énfasis en el aspecto más superficial y externo de las conductas puede traer consigo ante todo que se favorezcan las soluciones, y sobre todo las motivaciones, más fáciles y cómodas, que no son siempre las mejores; también que, en consecuencia, se vayan agotando progresivamente las fuentes más notables o elevadas, menos egoístas, para nuestra actuación; y asimismo que esta sea menos humana y personal, más dependiente del exterior, o heterónoma, e, incluso, que sea más hipócrita, si faltan esas fuentes o fundamentos más nobles para la actuación, pero se los sigue presuponiendo, porque siguen teniendo prestigio social o público, cuando en realidad es posible que ya no se tengan, sino que tan solo se los simule o aparente. Que esto sea de gran importancia también para la política, y en especial para la democracia moderna o representativa, donde, aparte de los controles jurídicos y políticos, hay que contar con un mínimo imprescindible de confianza en las personas que actúan como nuestros representantes, parece obvio. Aun cuando tampoco se puede negar la importancia que en ese terreno tiene la formación de un ambiente, o de una «cultura pública», que presione a favor de los comportamientos colectivamente deseados, y que a veces no se puede, o no conviene exigir por los procedimientos propiamente jurídicos, sino tan solo de ese modo más difuso y desorganizado.

Parece, pues, indudable que, por la trascendencia que tiene en múltiples aspectos, es conveniente procurar la diferenciación, al menos teórica o doctrinal, de los diversos sectores y conceptos. Esto lo echamos de menos en la obra de Rawls ${ }^{27}$; y, en la medida de nuestras fuerzas, hemos tratado de contrarrestarlo, al menos poniéndolo de manifiesto, aun cuando reduciéndolo también a los que consideramos sus verdaderos límites o términos.

${ }^{27}$ Así se expresa también J. Habermas, Faktizität und Geltung: Beiträge zur Diskurstheorie des Rechts und des demokratischenRechtsstaats, Frankfurt/M. (Suhrkamp), 1992, págs. 88-89 y 106. En cambio, no parece ser ésta la postura de los que entre nosotros escriben inspirándose en Raw $1 \mathrm{~s}$ o sobre él. Así, entre los primeros, creo que acentúa la indiferenciación de los diversos sectores y parece reducir la justicia al Derecho V. Camps, Virtudes públicas, Madrid (Espasa-Calpe), 1990: tampoco parece haber echado de menos la diferenciación A. Cortina, «La justificación ética del Derecho como tarea prioritaria de la filosofía política. Una discusión desde John Rawls», en Doxa, 2 (1985), págs. 129-144, y en Ética mínima, Madrid (Tecnos), 1992, págs, 177-204. 
